\title{
2021: a new year for the WHO
}

\author{
The United Nations Assembly has named 2021 the International Year of Peace and Trust. In that spirit, the world \\ can look forward with hope to the international collaborations spearheaded by the World Health Organization and \\ consider the challenges the agency has yet to face.
}

( n 20 January 2021, US

President-elect Joseph R. Biden Jr. will be sworn in as the 46 th president of the USA. At the top of his agenda, he has stated that the USA will rejoin the World Health Organization (WHO), from which President Donald Trump announced its withdrawal in May 2020, citing concerns about the WHO's response to the COVID-19 pandemic and its being unduly influenced by China. If unimpeded, the US withdrawal would precipitate a funding gap, a break in scientific interchange and, potentially, an unintended power vacuum amid the existing hurdles facing the WHO this year.

The anticipated departure of the USA exposed one of the agency's key vulnerabilities: sustainability of its funding. Assessed contributions by member states account for only $20 \%$ of the WHO budget, while voluntary contributions-such as those from the USA, its top donor-support $80 \%$ of its budget, which makes the WHO highly susceptible to the sudden loss of a major contributor. However, the timely formation of the WHO Foundation, also announced in May 2020, aims to address this fundamental weakness. This foundation will act as an independent body to raise and grant funds separately from the WHO, which will thereby enable it to tap into a broader corporate and individual donor base, lessen potential and perceived conflicts of interest, and strengthen the predictability of the WHO's funding. With a target of raising US $\$ 1$ billion in donations by 2023 , initially earmarked to address pandemic response and emergencies, the foundation has as its ultimate objective supporting the WHO's commitment to the United Nations Sustainable Development Goal 3 of health and well-being for all.

With financial security in sight, the agency must now work quickly to engage its partners to stem 2020's losses in key programmatic goals. Advances in vaccination against polio, the diagnosis and treatment of tuberculosis (TB), and interventions in malaria are predicted to have suffered enormous setbacks due to the effects of the pandemic lockdowns on access to healthcare, and to repurposing of funds and resources.

Some of these challenges predate the pandemic. In August 2020, eradication of polio reached a milestone with the certification of the WHO African Region as free of wild poliovirus, as it had no cases over the previous four years. Yet in Afghanistan and Pakistan, where the wild virus has never been eradicated, poliovirus infections have been rising, and strains of vaccine-derived poliovirus continue to spread in these countries, as well as in sub-Saharan Africa and in Southeast Asia. But the pandemic has exacerbated this burden, and pauses in polio-vaccination campaigns in 2020 are linked to an estimated 80 million unvaccinated children, which has necessitated renewed efforts to prevent large-scale outbreaks.

Similarly, at the end of 2019, most WHO regions were not anticipated to meet their 2020 TB milestones as defined in the WHO End TB Strategy. These include goals to reduce global TB incidence between 2015 and 2020 by $20 \%$, and TB deaths by $35 \%$, for which cumulative reductions of only $9 \%$ and $14 \%$, respectively, were achieved. Annual global funding of US\$900 million for TB research also fell far short of the US $\$ 2$ billion the WHO considers necessary to meet the End TB goals. Across the board, targets for increasing the diagnosis and treatment of $\mathrm{TB}$ and access to healthcare have not been met, and WHO modeling predicts that COVID19 may add an additional 1 million TB cases each year until 2025.

The situation for the control and treatment of malaria is equally daunting. The WHO 2020 global targets for malaria incidence and mortality fell short by $37 \%$ and $22 \%$, respectively, and the disruption of access to antimalarial treatment and services due to the COVID-19 pandemic is predicted to lead to tens of thousands additional deaths in sub-Saharan Africa.
Yet while the WHO has enormous challenges ahead to restore the gains in some of its core infectious-disease programs, there is hope on the horizon. In November 2020, the WHO approved, under its emergency use procedure, the deployment of the novel oral vaccine against polio, nOPV2, to curb the spread of vaccine-derived polioviruses. This precedent also sets the stage for the rapid dissemination of vaccines against COVID-19.

And through novel initiatives, the WHO is making headway in other areas of human health. In November 2020, the WHO launched the Global Strategy to Accelerate the Elimination of Cervical Cancer, its first campaign to eradicate a cancer. The initiative's goals include vaccination of $90 \%$ of girls against human papillomavirus by age 15 , and enabling access to treatment of $90 \%$ of women diagnosed with cervical disease by 2030 . A longitudinal population study of the efficacy of the vaccine against human papillomavirus in preventing invasive cervical cancer in girls and young women in Sweden supports the potential for success in this effort.

By renewing its ties to the USA and strengthening its finances, the WHO can better position itself to counter some of the losses to existing programs incurred by the COVID-19 pandemic. The WHO Director-General's Youth Council, tasked with providing advice on health and developmental issues specific to youth, as well as input on global health, is one such strategy for bringing new ideas to the table by the very group that will bear the long-term consequences of both the pandemic and today's health policy decisions. But 2021 also brings conceptual challenges to the WHO, and it will be a year for delineating the WHO's role on the global stage, restoring trust in the objectivity of its decisions, and driving collaboration of its member states to achieve the unifying goal of improving human health.

Published online: 13 January 2021

https://doi.org/10.1038/s41591-020-01213-5 